\title{
Path-Loss Estimation for Wireless Cellular Networks Using Okumura/Hata Model
}

\author{
Ibrahim Mohamed \\ Electrical and Electronic Department, Faculty of Engineering, Omar Al-Mukhtar University, Al-Beida, Libya
}

Email address:

engibrahim_2007@yahoo.com

\section{To cite this article:}

Ibrahim Mohamed. Path-Loss Estimation for Wireless Cellular Networks Using Okumura/Hata Model. Science Journal of Circuits, Systems and Signal Processing. Vol. 7, No. 1, 2018, pp. 20-27. doi: 10.11648/j.cssp.20180701.13

Received: November 30, 2017; Accepted: December 9, 2017; Published: January 11, 2018

\begin{abstract}
In cellular networks, accurate path-loss estimation is highly desirable not only to improve the performance but also to realize precise estimation of financial feasibility. In other words, while accurate path-loss estimation helps to achieve an acceptable performance and reasonable cost, inaccurate path-loss estimation would either lead to degrade the performance, or increase the cost. Different models were introduced in the literature for achieving accurate path-loss estimation. One of theses models is the Okumura/Hata model which is recommended for being used to estimate the path-loss in the cellular systems that employing micro-cells. It is characterized by its suitability for being used in a variety of environments. The main objective in this paper is to provide a guide line for path-loss estimation analysis using Okumura/Hata model. Matlab software was used to perform this analysis. Compared with free space model in which frequency and separation distance are the only contributors for path-loss, more accurate estimation can be achieved when Okumura/Hata model is used as it includes further correction factors, such as mobile station antenna height and base station antenna height.
\end{abstract}

Keywords: Cellular Networks (CNs), Path-Loss Estimation Models, Free Space Propagation Model, Okumura/Hata Model

\section{Introduction}

One of the main objectives of cellular systems is to achieve high capacity (increase the number of user). This objective can be realized by creating a distinctive way by which the limited frequency spectrum assigned to the cellular systems is exploited efficiently. The concept of frequency reuse in which a segment of specific frequency spectrum can be used several times is the key to achieve an efficient exploit of the assigned spectrum. In frequency re-use theory, each cell within a single cluster represents a replica of one of six adjacent cells (co-channel cells) surrounding it. Each cochannel cell belongs to a neighbor cluster. Although a lowpower transmitter is commonly employed in each cell, a sufficient distance should be determined for co-channel cells separation to guarantee minimum interference. Although interference can also come from the second and higher tires co-channel cells, such interference can be ignored as it often contributes by less than one percent $(1 \%)$ of the total interference [1]. As a type of communication system, the performance of a cellular system is indicated by its signal-tonoise ratio $S N R$. While the term signal refers to the power of an intended carrier, noise represents the power produced at the receiver due to thermal effect plus the sum of the unwanted but received powers produced by the co-channel cells. For acceptable performance, a cellular system should meet a specific value of $S N R$. SNR may differ from system to another. For example, approximately, a minimum $S N R$ value of $18 d B$ or $12 d B$ is specified for acceptable performance when advanced mobile phone system AMPS or global system for mobile communication GSM is considered, respectively [2]. In cellular systems, a wireless channel is mainly characterized by its effects of dispersion and attenuation. While dispersion is analyzed to verify the kind of signal distortion encountered during propagation, and thus take the proper solution to minimize it, attenuation is analyzed to estimate the path-loss. Dispersion can be categorized as either time-based dispersion or frequency-based dispersion. In a wireless channel, a signal might be attenuated due to different phenomena, such as reflection, diffraction, or scattering. Reflection occurs when a signal that is propagating in a medium passes to another medium with different properties. Attenuation in this case can be attributed to that a part of the incident signal energy is either absorbed 
or propagated into the reflecting medium. Diffraction is defined as the deviation of a signal from its path. It occurs when a signal that is propagating in a medium passes into a shadow region created by an obstruction. Scattering is defined as the spreading of energy out of its intended path. It occurs when a signal hits an object whose size is much smaller than or on the order of the signal wavelength. Accurate path-loss estimation is highly recommended in the initial stages of cellular network design to precisely determine the number of cell sites required for providing coverage in a given area, which leads to achieve a simple estimation of financial feasibility. Additionally, accurate path-loss estimation can help to realize an acceptable system performance. Figure 1(a), (b), and (c) clarifies the aforementioned situations in which the estimated path-loss is almost equal to the actual path-loss in (a), lower than the actual path-loss in (b), or higher than the actual path-loss in (c). Moreover, accurate path-loss estimation can help to achieve an optimum cell site location [2].

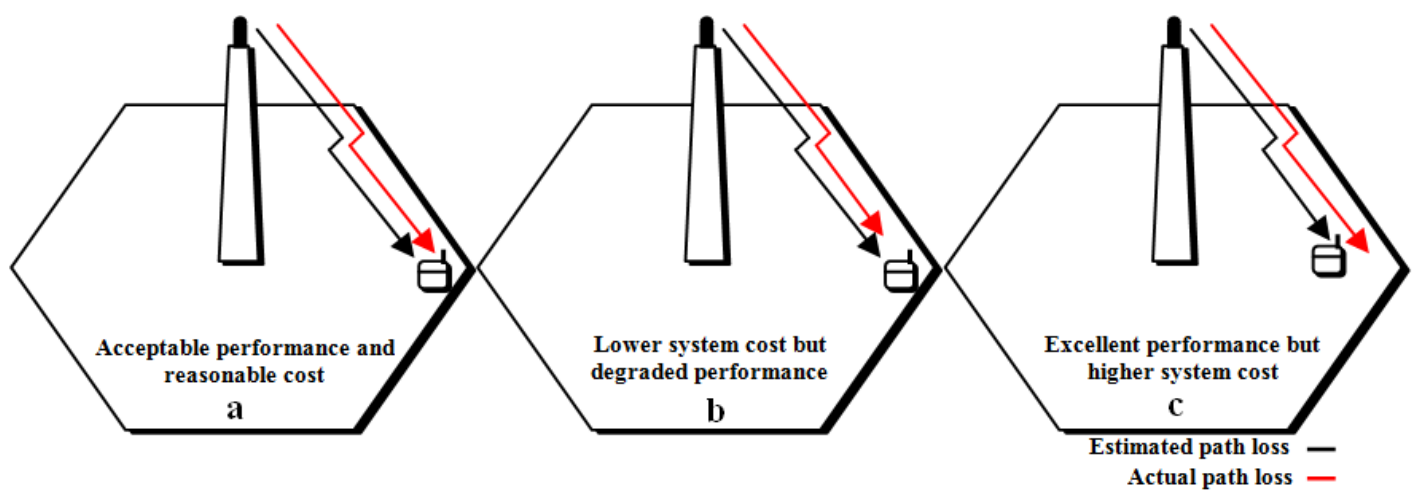

Figure 1. Effect of accurate and inaccurate path-loss estimation.

Different models were introduced in the literature for achieving accurate path-loss estimation [4] [5] [6] [7] [8]. Path-loss models can be categorized according to the separation distance as either long-distance prediction models intended for macro-cells or short-distance prediction models intended for micro-cells. Pico-cells (cells that cover part of a building and mainly span from 30 to 100 meters) can be included in the short-distance prediction models. Indoor prediction models were introduced to estimate the path-loss in this case. Figure 2 provides a diagrammatic categorization of the path-loss models. They started with the simplest lineof-sight path-loss model; also referred to as free space propagation model. In free space propagation model, no obstructions due to earth surface or other obstacles are encountered during propagation. Among these models is the Okumura/Hata model which is characterized by its suitability for being used in a variety of non-line-of-sight environments (i.e. typical urban, typical suburban, and rural environments).

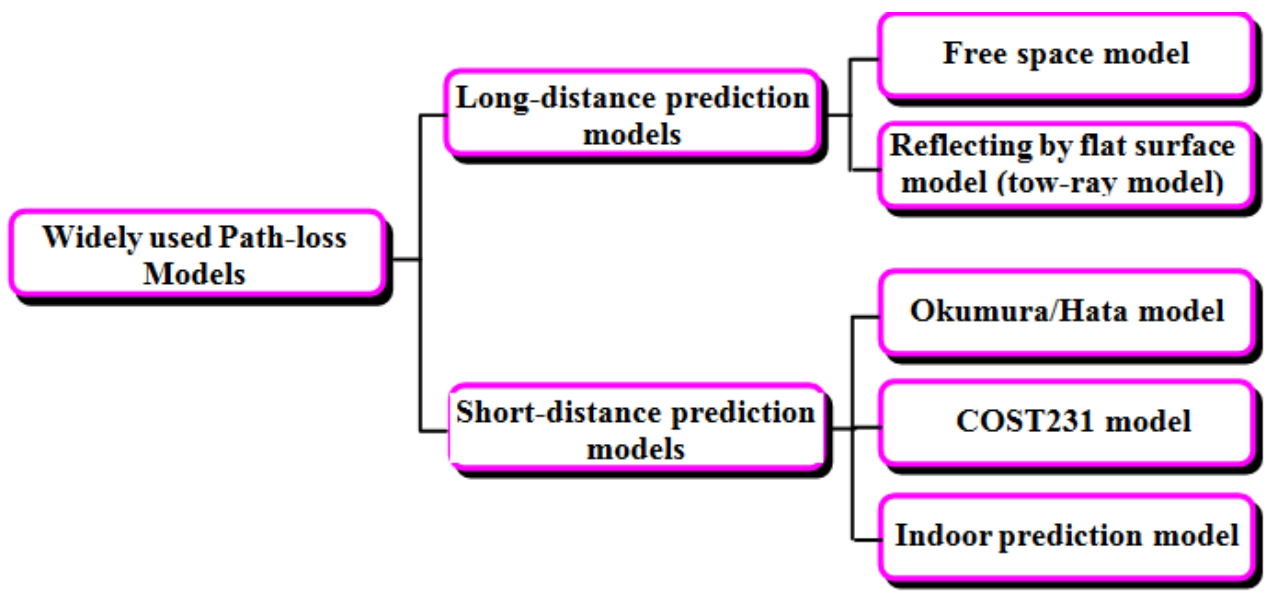

Figure 2. Widely used path-loss models.

Unlike free space model in which frequency and separation distance (distance between transmitter and receiver) are the only contributors used for estimating the path-loss, further factors such as mobile station antenna height and base station antenna height are included in the Okumura/Hata model to achieve more accurate path-loss estimation. In this paper, the author aims to provide a guide line for cellular network designers and operators to estimate the path-loss using Okumura/Hata model. This paper is organized as follows: Section 2 provides theoretical background of Free-space path-loss estimation model and Okumura/Hatapath-loss estimation model. Results are discussed in section 3. Section 4 concludes the paper. 


\section{Theoretical Background}

In cellular networks, accurate path-loss estimation helps to precisely determine the number of cell sites required for providing coverage in a given area. While accurate path-loss estimation leads to realize an acceptable system performance and achieve reasonable cost estimation, inaccurate path-loss estimation would either lead to degrade the system performance or increase the cost. Various path-loss estimation models were introduced in the literature; each of which aiming to achieve accurate path-loss estimation. In this section, theoretical background of free-space path-loss estimation model and Okumura/Hata path-loss estimation model is provided.

\subsection{Free-Space Path-Loss Estimation Model}

Free space model is one of the well-known long-distance prediction models. In free space model, a line-of-sight propagation path is assumed. i.e., no obstructions exist between transmitter and receiver albeit by the surface of earth. It is mostly applicable for wireless channels in which a transmission system such as microwave or satellite-tosatellite is employed. The path-loss in this case is referred to as free space path-loss ( $\left.L_{P f r e e}\right)$. Free space path-loss is given as [9]

$$
L_{\text {Pfree }}=\left[\frac{4 \pi d}{\lambda}\right]^{2} \text { where } d \text { and } \lambda \text { represent the separation }
$$
distance (i.e., distance between the transmitter and receiver) and wavelength, respectively. Given $\lambda=c / f$ yields $L_{\text {Pfree }}=\left[\frac{4 \pi f d}{c}\right]^{2}$ where $c$ and $f$ represent the speed of light $\left(3 \times 10^{8} \mathrm{~m} / \mathrm{sec}\right)$ and frequency in hertz $\mathrm{Hz}$, respectively. Substituting $d$ in kilometer and $f$ in megahertz, free space path-loss can be expressed in $d B$ as

$$
L_{\text {Pfree }[d B]}=32.44+20 \log f+20 \log d
$$

It can be observed from (1) that duplication of frequency or distance would lead an increase in the path-loss by $6 \mathrm{~dB}$.

\subsection{Okumura/Hata Model}

Okumura/Hata model is one of the well-known non-loneof-sight models. It was primarily developed for path-loss estimation in typical flat urban environment. Then it was improved to include path-loss estimation in the typical suburban and rural environments. A correction factor that is related to the mobile station antenna height was included in different forms according to the environment considered and frequency carrier used to achieve accurate path-loss estimation. The path-loss in $d B$ according to Okumura/Hata model for typical urban environment is given as [9] [10]

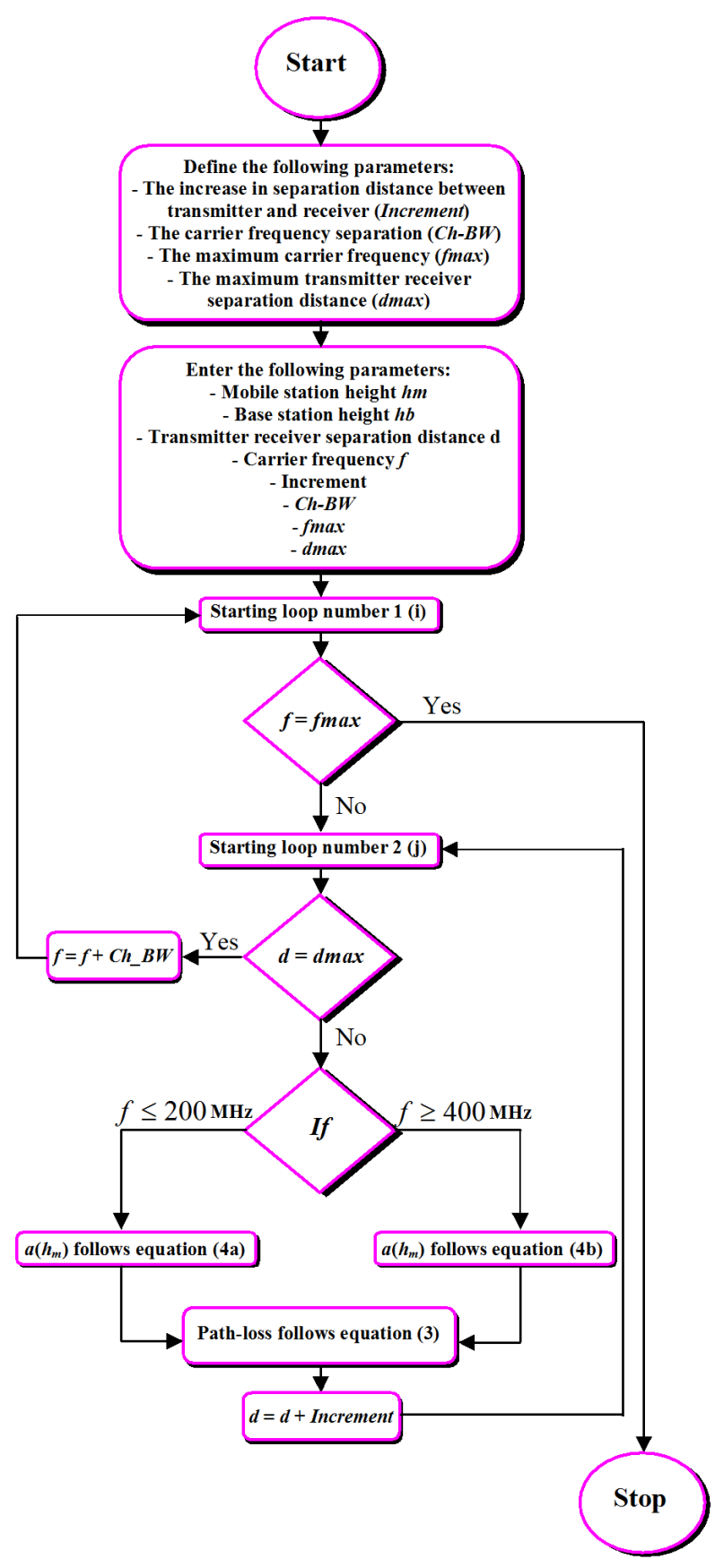

Figure 3. Sequence of the program operation where typical urban environment Okumura/Hata model for large cities is considered.

$$
L_{50}(\text { urban })_{[d B]}=69.55+26.16 \log f c+\left(44.9-6.55 \log h_{b}\right) \log d-13.82 \log h_{b}-a\left(h_{m}\right)
$$

Where $f_{c}$ is the carrier frequency in $M H z, d$ is the separation distance between the mobile station and base station in $K m, h_{b}$ and $h_{m}$ are the base station and mobile station antennas height in meter, and $a\left(h_{m}\right)$ is correction factor that is related to the mobile station antenna height. $a\left(h_{m}\right)$ is given in different forms according to the size of the intended area. For large cities and at $f_{c} \leq 200 \mathrm{MHz}, a\left(h_{m}\right)$ is given as 


$$
a\left(h_{m}\right)=8.29\left[\log \left(1.54 h_{m}\right)\right]^{2}-11
$$

For large cities and at $f_{c} \geq 400 \mathrm{MHz}, a\left(h_{m}\right)$ is given as

$$
a\left(h_{m}\right)=3.2\left[\log \left(11.75 h_{m}\right)\right]^{2}-4.97
$$

For small and medium-sized cities

$$
a\left(h_{m}\right)=\left[1.1 \log \left(f_{c}\right)-0.7\right] h_{m}-\left[1.56 \log \left(f_{c}\right)-0.8\right]
$$

The path-loss in $d B$ according to Okumura/Hata model for the typical suburban environment is given as

$$
L_{50}(\text { suburban })_{[d B]}=L_{50}(\text { urban })-2\left[\left(\log \left(\frac{f c}{28}\right)^{2}\right)-5.4\right]
$$

The path-loss in $d B$ according to Okumura/Hata model for the rural environment is given as

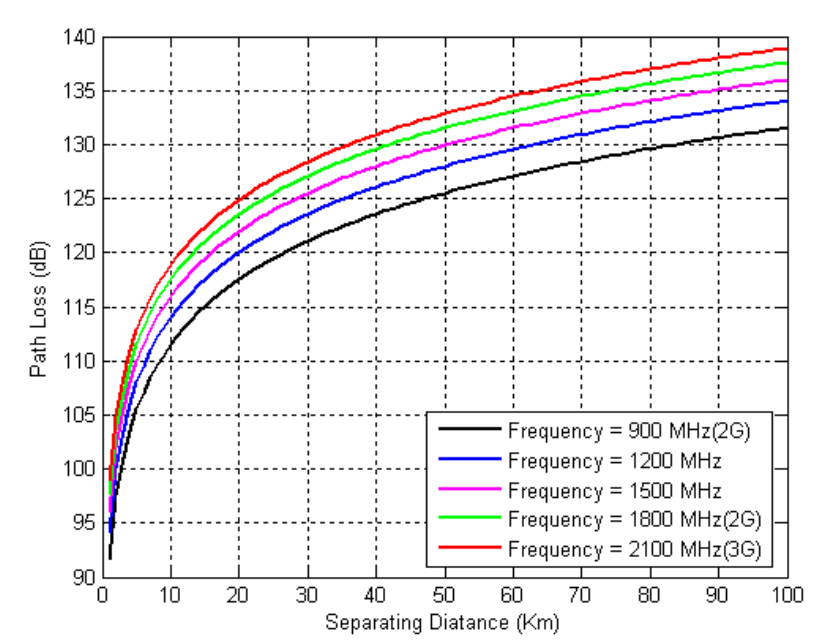

Figure 4. Path-loss vs separating distance at different carrier frequencies: Free space model was considered.

$$
L_{50}(\text { rural })_{[d B]}=L_{50}(\text { urban })-4.78(\log f \mathcal{c})^{2}+18.33 \log f c-40.94
$$

Table 1 provides the range of parameters for which Okumura/Hata model is valid [9].

Table 1. The range of parameters for which Okumura/Hata model is valid.

\begin{tabular}{lll}
\hline & Minimum value & Maximum value \\
\hline Carrier frequency $f_{c}(\mathrm{MHz})$ & 150 & 2200 \\
Base station height $h_{b}(\mathrm{~m})$ & 30 & 200 \\
Mobile station height $h_{m}(\mathrm{~m})$ & 1 & 10 \\
Separation distance $d(\mathrm{Km})$ & 1 & 20 \\
\hline
\end{tabular}

Going through details of sections 2.2, one can note that it might be needed to perform a long and complex calculation approach to estimate the path-loss when Okumura/Hata model is used. To avoid complexity in calculations and save the time, the approach we used to estimate the path-loss was by composing MATLAB computing programs and involving the loop mechanism in these programs. Figure 3 is a sampled flowchart describing the sequence of program operation.

\section{Results and Discussion}

This section is divided into two subsections. Results that estimate the path-loss for Free-space path-loss prediction models were provided in the first subsection whereas; results that estimate the path-loss estimations for Okumura/Hata prediction model were provided in the second subsection. In both subsections, graphical representations of path-loss versus separation distance at different effective factors were provided. In all cases, path-loss at any intended distance can be readily estimated by drawing a vertical line at that distance such that it intersects the path-loss versus distance curve and then draw a horizontal line from the intersection point; the value of path-loss in this case is the intersection point with the path-loss axis. Carrier frequencies of 900 $\mathrm{MHz}, 1800 \mathrm{MHz}$, and $2100 \mathrm{MHz}$ were selected in the analysis as they represent the carrier frequencies used in the GSM standard in its forms of $2^{\text {nd }}$, and $3^{\text {rd }}$ generations cellular networks, respectively. The Matlab software (The Mathworks, Inc., Natick, MA, USA) was used for realizing the graphical representation.

\subsection{Path-Loss Estimations Considering Free-Space Model}

In this part of results, the path-loss is estimated by considering equations (1). Figure 4 shows the graphical representation of (1). It represents the path-loss vs separating distance at different carrier frequencies, where free space model is considered. It can be obviously seen from the graph that the path-loss starts to appear at a certain value of separation distance and continues to increase rapidly with a slight increase in the separation distance. However, it starts to increase steadily at higher values of separating distance. It can be obviously observed from the graph that the smallest value of path-loss is encountered when the lowest carrier frequency is assigned.

\subsection{Path-Loss Estimation Considering Okumura/Hata Model}

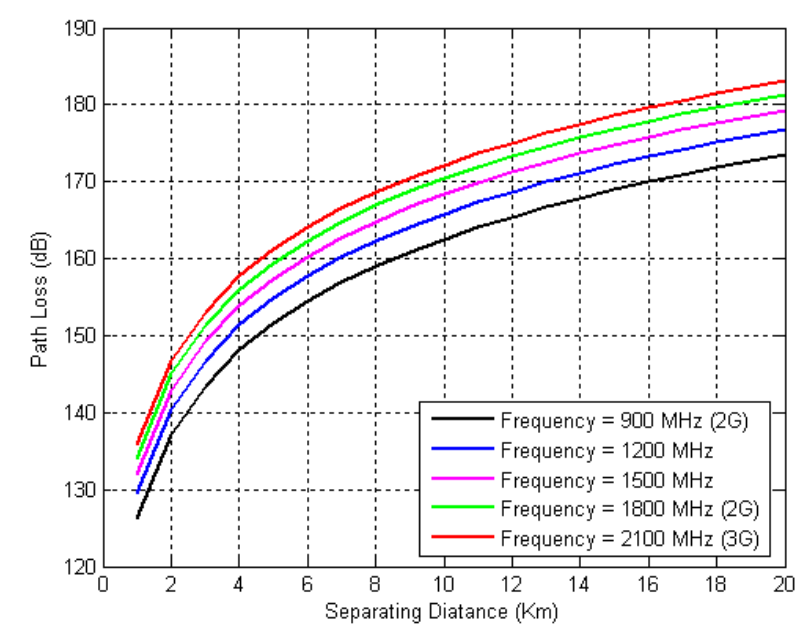

Figure 5. Path-loss vs separating distance at different carrier frequencies: typical urban environment Okumura/Hatta model for large cities is considered. 
In this part of results, the path-loss is estimated by considering equations (2) to (7). Graphical representation of the path-loss versus separation distance $(1 \mathrm{Km}$ to $20 \mathrm{Km})$ was provided. Further graphical representations that include significant parameters which might affect the path-loss estimation, such as carrier frequency $(900,1800$, and $2100 \mathrm{MHz})$, and base station height $(20,80,140$, and $200 \mathrm{~m})$ were provided. Figure 5 shows the graphical representation of equations (2), (3) and (4). It represents the path-loss vs separating distance at different carrier frequencies, where the typical urban environment Okumura/Hata model for large cities is considered. It can be obviously seen from the graph that the path-loss starts to appear at a certain value of separating distance in which the model is valid $(1 \mathrm{Km})$ and continues to increase with the increase of the separating distance.

Figure 6 ( $a, b$ and $c)$ shows the path-loss versus separating distance at different values of base station heights where typical urban environment Okumura/Hata model for large cities is considered. It can be obviously seen from the graph that the path-loss starts to appear at a certain value of separating distance in which the model is valid $(1 \mathrm{Km})$ and continues to increase with the increase of the separating distance. However, an increase in the base station height could lead to reduce the path-loss. Figure 7 shows the graphical representation of equations (2) and (5). It represents the path-loss vs separating distance at different carrier frequencies, where the typical urban environment Okumura/Hata model for small and mid-sized cities is considered. It represents the path-loss vs separating distance at different carrier frequencies, where the typical urban environment Okumura/Hata model for small and mid-sized cities is considered. The graph follows the same behavior as in Figure 5 with a slight difference can be observed. For example, a path-loss value of $183.1184 d B$ was measured at $20 \mathrm{Km}$ separation and $2100 \mathrm{MHz}$, when typical urban environment Okumura/Hata model for large cities was considered whereas a path-loss of $181.9678 d B$ was measured at an identical separation distance and frequency carrier when typical urban environment Okumura/Hata model for small and mid-sized cities was considered.

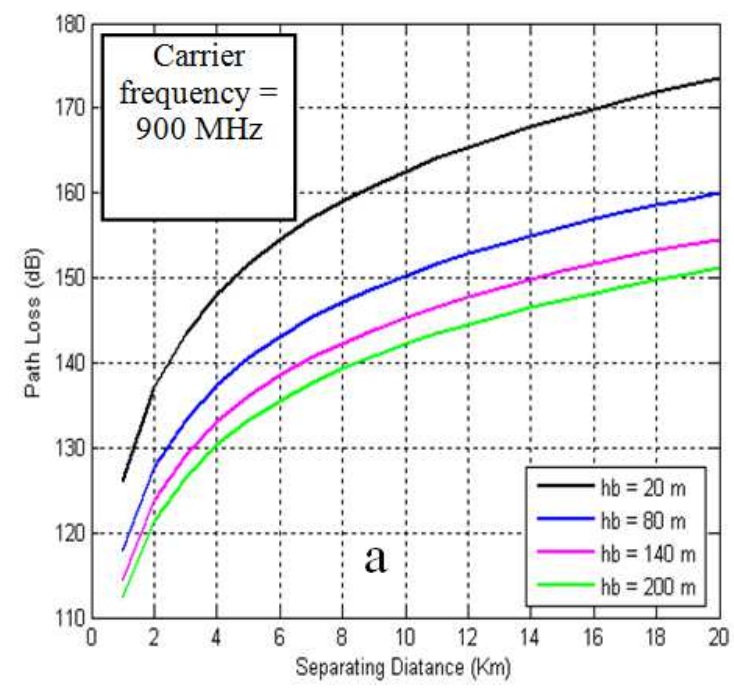

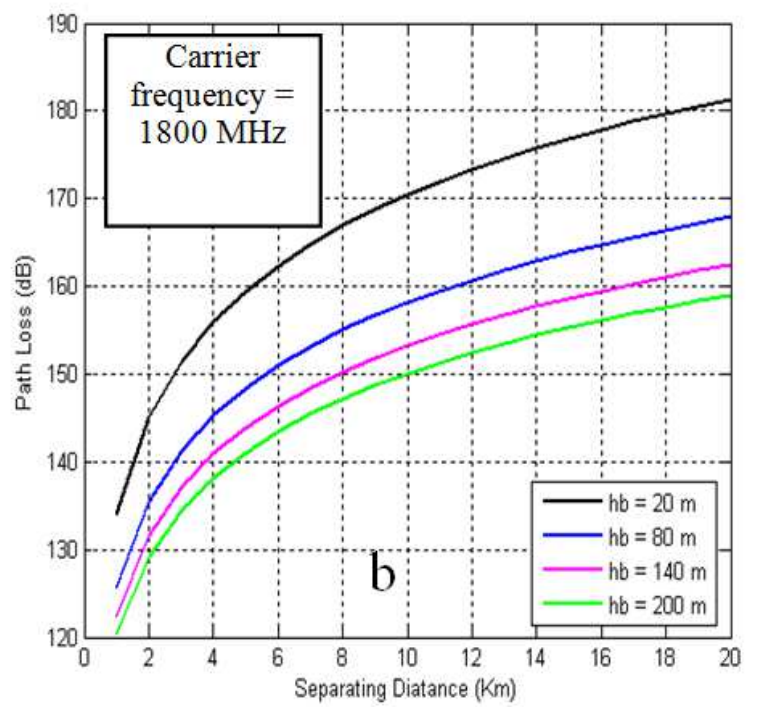

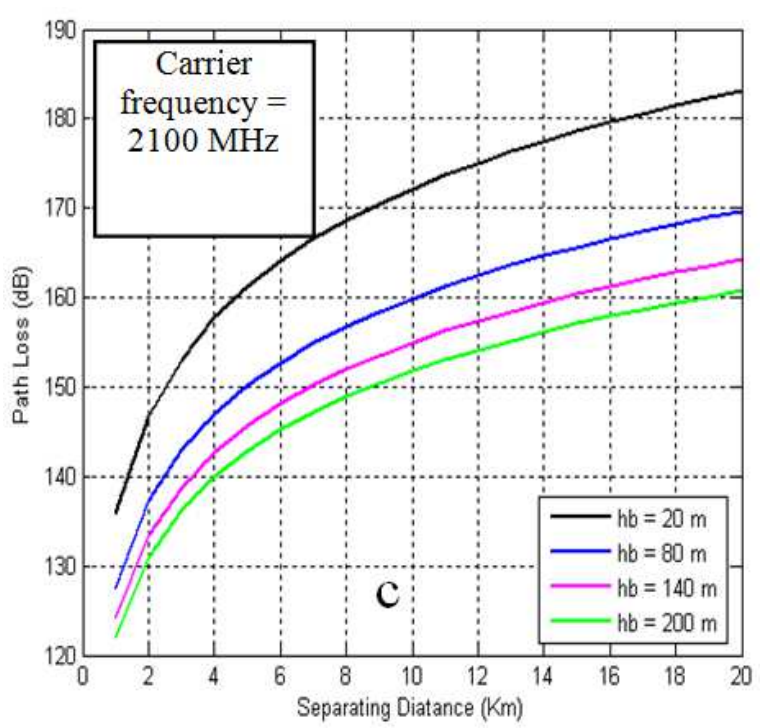

Figure 6. Path-loss versus separating distance at different base station heights: Okumura/Hatta model for typical urban environment was considered.

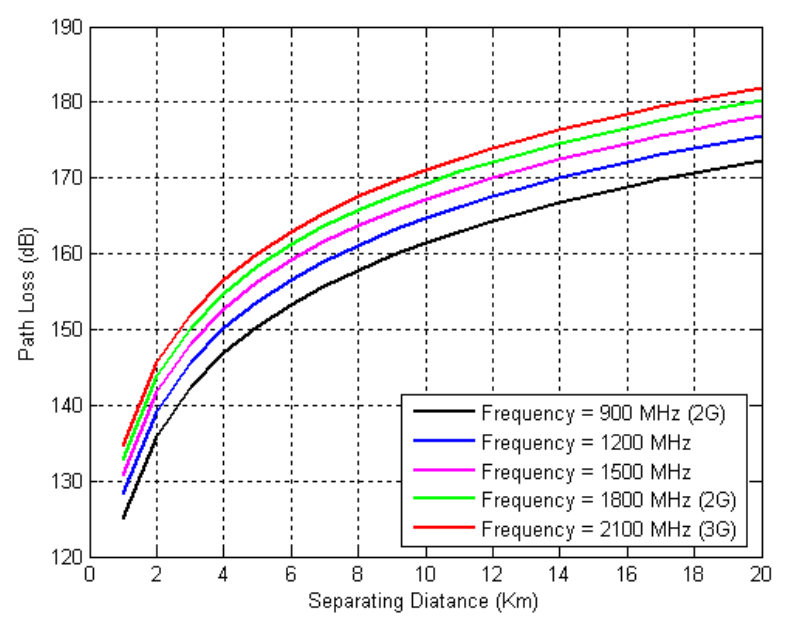

Figure 7. Path-loss vs separating distance at different carrier frequencies: typical urban environment Okumura/Hatta model for small and mid-sized cities is considered. 

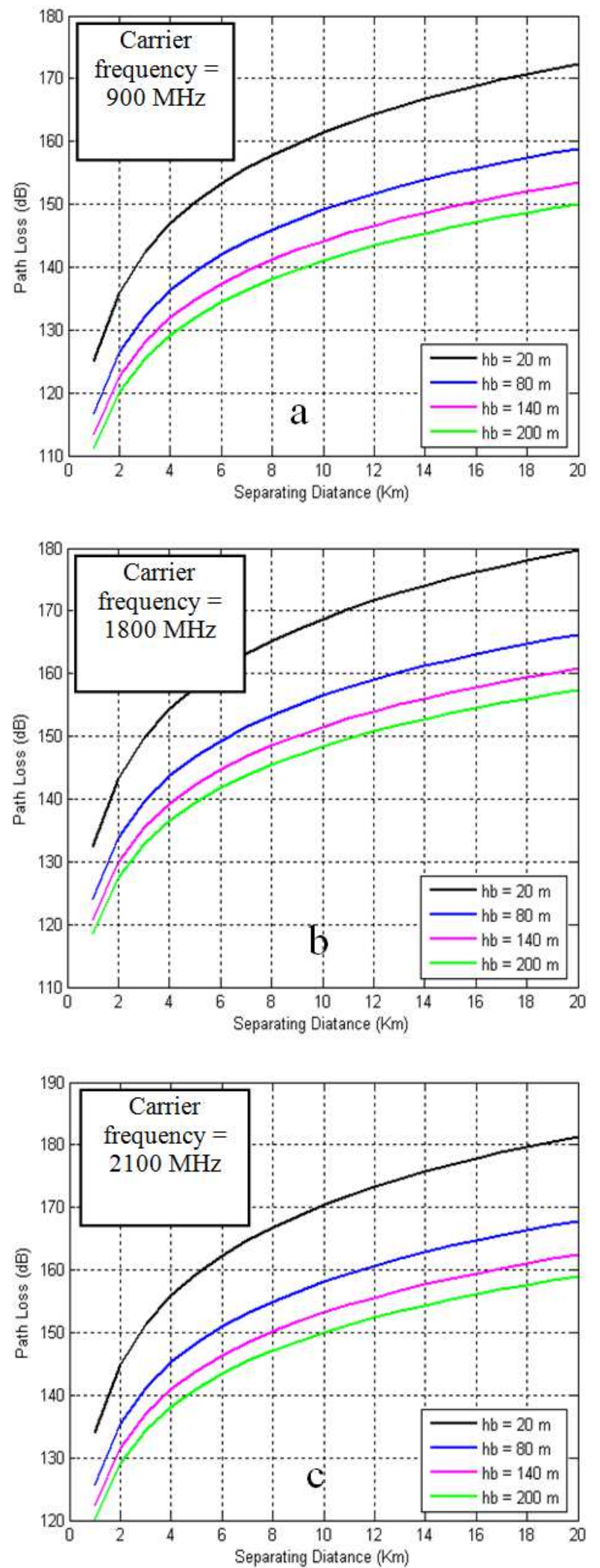

Figure 8. Path-loss versus separating distance at different base station heights: Okumura/Hatta model for typical urban environment was considered.

Figure $8(\mathrm{a}, \mathrm{b}$ and $\mathrm{c})$ shows the path-loss versus separating distance at different values of base station heights where typical urban environment Okumura/Hata model for small and mid-sized cities is considered. The graph follows the same behavior as in Figure 6 with a slight reduction in the path-loss can be observed. For example, a path-loss value of 158.9858 $d B$ was measured at $200 \mathrm{~m}$ base station height, 20 $\mathrm{Km}$ separation, and $2100 \mathrm{MHz}$ when typical urban environment Okumura/Hata model for small and mid-sized cities was considered whereas a path-loss of $160.7766 \mathrm{~dB}$ was measured at an identical base station height, separation distance, and frequency carrier when typical urban environment Okumura/Hata model for large cities was considered. Figure 9 shows the graphical representation of equation (6). It represents the path-loss vs separating distance at different carrier frequencies, where the typical suburban environment Okumura/Hata model is considered. The graph follows the same behavior as in Figures 5 and 7 with a slight difference can be observed. For example, a path-loss value of $185.2676 \mathrm{~dB}$ was measured at $20 \mathrm{Km}$ separation and 2100 $\mathrm{MHz}$, when typical suburban environment Okumura/Hata model was considered whereas a path-loss of $181.9678 \mathrm{~dB}$, or $183.1184 d B$ were measured at an identical separation distance and frequency carrier when typical urban environment Okumura/Hata model for small and mid-sized cities, or typical urban environment Okumura/Hata model for large cities were considered, respectively.

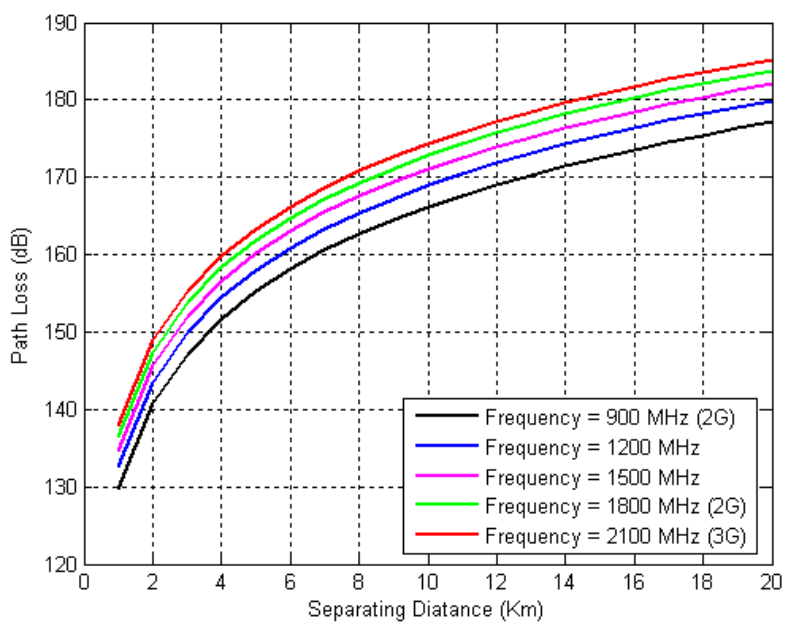

Figure 9. Path-loss vs separating distance at different carrier frequencies: typical suburban environment Okumura/Hatta is considered.

Figure 10 (a, b and c) shows the path-loss versus separating distance at different values of base station heights where typical suburban environment Okumura/Hata model is considered. The graph follows the same behavior as in Figures 6 and 8 with a decrease in the path-loss can be observed. For example, a path-loss value of $154.7715 d B$ was measured at $200 \mathrm{~m}$ base station height, $20 \mathrm{Km}$ separation, and $2100 \mathrm{MHz}$ when typical suburban environment Okumura/Hata model was considered whereas a path-loss of $158.9858 d B$ or $160.7766 d B$ were measured at an identical base station height, separation distance, and frequency carrier when typical urban environment Okumura/Hata model for small and mid-sized cities or typical urban environment Okumura/Hata model for large cities were considered. 

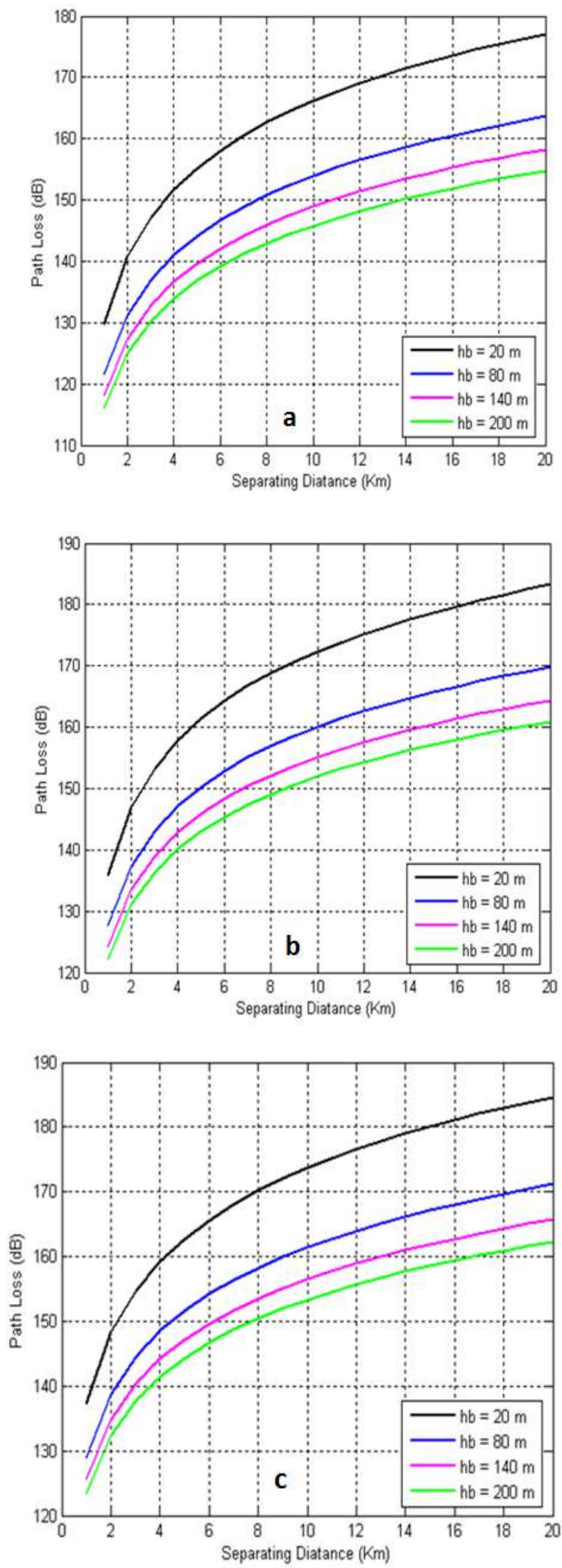

Figure 10. Path-loss versus separating distance at different base station heights: Okumura/Hatta model for typical suburban environment was considered.

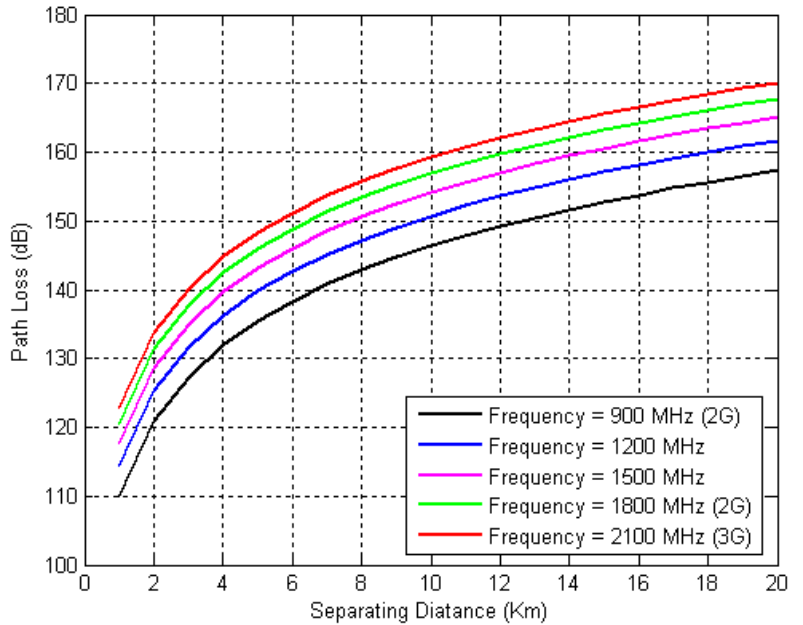

Figure 11. Path-loss vs separating distance at different carrier frequencies: rural environment Okumura/Hatta is considered.
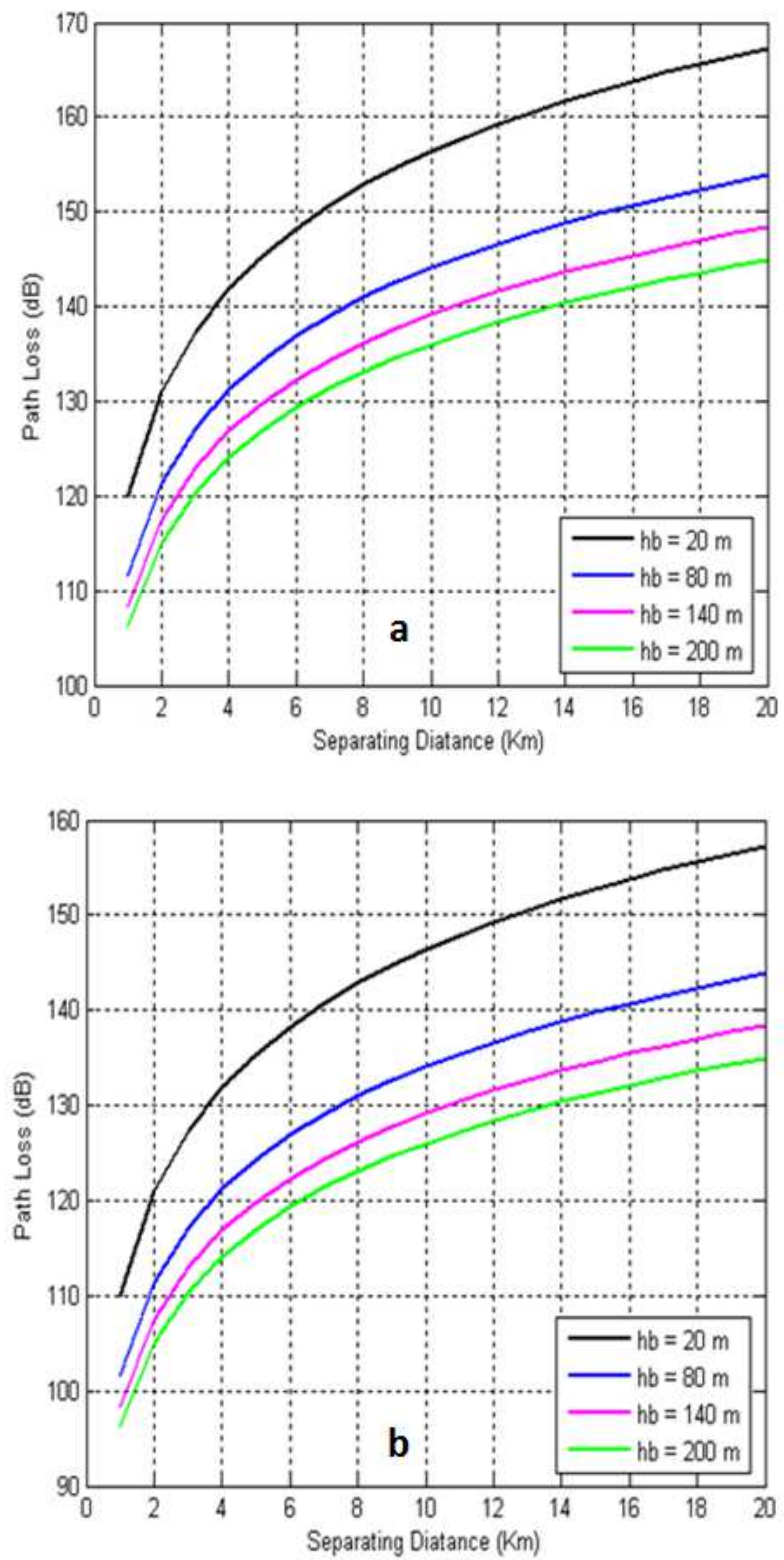


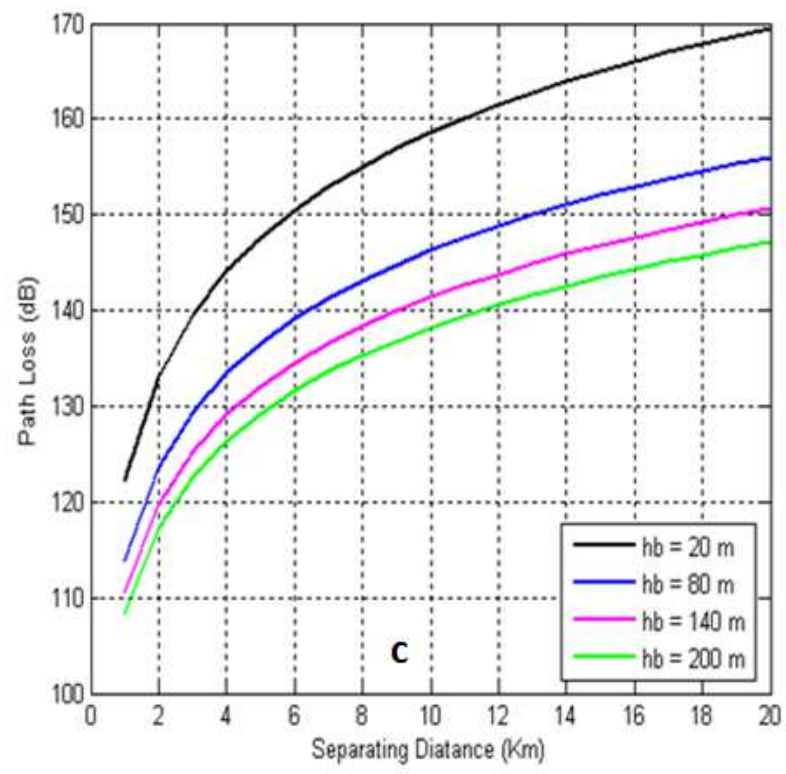

Figure 12. Path-loss versus separating distance at different base station heights: Okumura/Hatta model for rural environment was considered.

Figure 11 shows the graphical representation of equation (7). It represents the path-loss vs separating distance at different carrier frequencies, where the rural environment Okumura/Hata model for is considered. The graph follows the same behavior as in Figures 5, 7 and 9. However a notable decrease in the path-loss can be observed. For example, a path-loss value of $170.1637 d B$ was measured at $20 \mathrm{Km}$ separation and $2100 \mathrm{MHz}$, when rural environment Okumura/Hata model was considered whereas a path-loss of $181.9678 d B, 183.1184 d B$, or $185.2676 d B$ were measured at an identical separation distance and frequency carrier when typical urban environment for small and mid-sized cities, typical urban environment for large cities, or typical suburban environment Okumura/Hata models were considered, respectively. Figure 12 (a, b and c) shows the path-loss versus separating distance at different values of base station heights where rural environment Okumura/Hata model is considered. The graph follows the same behavior as in Figures 6, 8, and 10. However a notable decrease in the path-loss can be observed. For example, a path-loss value of $147.1817 \mathrm{~dB}$ was measured at $200 \mathrm{~m}$ base station height, 20 $\mathrm{Km}$ separation, and $2100 \mathrm{MHz}$ when rural environment Okumura/Hata model was considered whereas a path-loss of $154.7715 d B, 158.9858 d B$, or $160.7766 d B$ were measured at an identical base station height, separation distance, and frequency carrier when typical suburban environment, typical urban environment for small and mid-sized cities, or typical urban environment for large cities Okumura/Hata models were considered, respectively.

\section{Conclusions}

A guide line for path-loss estimation using Okumura/Hata model was provided. Compared with free space model in which frequency and separation distance are the only contributors for path-loss, more accurate estimation can be achieved when Okumura/Hata model is used as it includes further correction factors, such as mobile station antenna height and base station antenna height. Going through results obtained, there were some significant observations that can be exploited advantageously. For example, results on Figures $6,8,10$, and 12 confirm that an increase in the base station height leads to reduce the path-loss. This can be exploited advantageously and thus give the opportunity to achieve a lower path-loss. However, much higher base station would affect the cost effectiveness of the cellular system. On other words, the base station height should be chosen such that it is comparable to the surrounding buildings.

\section{References}

[1] Jochen H. Schiller, Mobile Communications, PEARSON EDUCATION LIMITED, 2003

[2] Andrea Goldsmith, WIRELESS COMMUNICATIONS, Cambridge University Press, 2005

[3] Yoo-Seung Song and Hyun-Kyun Choi, Analysis of V2V Broadcast Performance Limit for WAVE Communication Systems Using Two-Ray Path Loss Model, ETRI Journal, Volume 39, Number 2, pp 213-221, April 2017

[4] Divya Kurup, Maria Scarpello, Günter Vermeeren, Wout Joseph, Kristof Dhaenens, Fabrice Axisa, Luc Martens, Dries Vande Ginste, Hendrik Rogier, Jan Vanfleteren, In-body path loss models for implants in heterogeneous human tissues using implantable slot dipole conformal flexible antennas, EURASIP Journal on Wireless Communications and Networking 2011

[5] Haipeng Ding, Zhengyuan Xu, Brian M. Sadler, A Path Loss Model for Non-Line-of-Sight UltravioletMultiple Scattering Channels, EURASIP Journal onWireless Communications and Networking Volume 2010, Article ID 598572, 12 pages

[6] Mario Versaci, Salvatore Calcagno, Fabio La Foresta, Biagio Cammaroto, PATH LOSS PREDICTION USING FUZZY INFERENCE SYSTEM AND ELLIPSOIDAL RULES, American Journal of Applied Sciences, Science Publication, 2012, 9 (12), 1940-1943

[7] A. Bhuvaneshwari, R. Hemalatha, T. Satyasavithri, Semi Deterministic Hybrid model for Path Loss prediction improvement, 2nd International Conference on Intelligent Computing, Communication \& Convergence (ICCC-2016), pp-336-344

[8] Yun-Jie Xu, Wen-Bin Li, Propagation path loss prediction model of multi-sensor network in forest, Advanced in Control Engineering and Information Science, pp-2206 - 2210, published by Elsevier, 2011

[9] Vijay K. Garg, Wireless Communications and Networking, Elsevier, 2007

[10] Kazunori Uchida; Naoto Hadano; Masafumi Takematsu; Junichi Honda, Propagation Estimation by Using Building Coverage and Floor Area Ratios Based on 1-Ray Model Combined with Okumura-Hata Mode, 17th International Conference on Network-Based Information Systems, 2014, pp: 555-560 